\title{
The Influence of Motion Path and Assembly Sequence on the Stability of Assemblies
}

\author{
Sourav Rakshit and Srinivas Akella \\ Department of Computer Science, University of North Carolina at Charlotte, USA \\ $\{$ srakshit,sakella\}@uncc.edu
}

\begin{abstract}
In this paper we present an approach for the stability analysis of mechanical part disassembly considering part motion in the presence of physical forces such as gravity and friction. Our approach uses linear complementarity to analyze stability as parts are moved out of the assembly. As each part is removed from the assembly along a specified path during disassembly, we compute the contact forces between parts in the remaining assembly; positive contact forces throughout the disassembly process imply the disassembly sequence is stable (since the parts remain in contact with one another). However, if the part that is being taken out induces motion of other parts in the remaining subassembly, we conclude the disassembly sequence is unstable. Thus, we are able to simulate the entire disassembly sequence considering physical forces and part motion, which has not previously been done. We then show the influence of part motion on the selection of stable disassembly sequences. In contrast to prior work on disassembly which has focused either on planning part motions based on only geometric constraints, or on analyzing the stability of an assembly without considering part motions, we explore the relation between part motion and the selection of stable disassembly sequences. Since we track the motion of all parts in an assembly, instability inducing motions can be identified and prevented by introducing appropriate fixtures, by selecting alternative disassembly sequences, or by changing the motion paths. We also describe when the presence of physical forces can make assembly and disassembly noninvertible, i.e., disassembly is not the inverse of assembly.
\end{abstract}

\section{INTRODUCTION}

Product assembly is a labor intensive and time consuming process $[25,5]$. Automated robotic assembly is aimed at reducing human cost and time. There has been significant research on several aspects of assembly automation including the development of algorithms for assembly sequencing considering part geometry [26], grasping of objects considering physical forces [15, 20], stability of subassemblies [17, 16, 18], and motion planning for part removal [23, 11, 14]. The simplest assembly problem is one in which there are only two hands, and the assembly sequence is monotone, i.e., each part is moved directly to its final position in the product without being placed in intermediate positions [26].

In this work we consider monotone assembly of rigid parts using two hands. A classical strategy for assembly planning is assembly-by-disassembly [13]. This strategy is popular in assembly planning since a product in its assembled state has many more constraints than in its disassembled state; these constraints reduce the search space for a planner. When the parts are rigid and when only geometric constraints are considered, an inverted disassembly sequence leads to a feasible assembly sequence. Thus the terms assembly and disassembly have been used interchangeably in the literature [26]. We make the initial assumption that a stable disassembly sequence when inverted will become a stable assembly sequence. In our work the fixed support is considered as a hand, so there is only one moving hand. The stability analysis we perform also has potential applications for assistive robots in domestic environments, where tasks like stacking books, dishes, boxes, and even blocks (e.g., in the game Jenga [24]) are common. It may also be useful for autonomous construction [10], and safe removal of collapsed structures during rescue operations after disasters such as earthquakes.

The focus of our work is the stability of the assembly in the presence of physical forces as each part is taken out of the assembly. We calculate the forces arising between the parts using linear complementarity [2, 22]. The part motions are selected using the non-directional blocking graph (NDBG) [26]. Our stability analysis is based on calculating the contact forces and identifying relative part motion. The initiation of breaking of contact in frictionless assemblies is reflected as a positive acceleration at the contact points, which is complementary to the contact force at that point of contact. For assemblies with friction, the relative motion is indicated by the sliding velocity. Thus we can simulate the stability of the disassembly process over time. Although the stability of an assembly has been analyzed previously [3, 17, 16, 18], and such analysis has been used to analyze the disassembly tree [19], the simulation of disassembly considering physical forces over time has not been previously addressed. Hence, our work can be incorporated into disassembly simulation and disassembly motion planning based on physical forces and constraints. Our analysis checks the stability of the assembly as the constituent parts are moved in the presence of physical forces such as gravity and friction. Further, we demonstrate that the stability of disassembly sequences is impacted by not only the disassembly sequence, but also by the motion paths taken by the parts. Thus in the presence of physical forces, two-handed monotone assembly and disassembly can be noninvertible.

\section{RELATED WORK}

There are two main areas of research related to our work: stability analysis, and geometric analysis of assemblies. However there has not been much prior work uniting these two areas for assembly planning. 


\section{A. Stability Analysis}

Blum et al. [3] first analyzed the stability of rigid blocks. They defined an assembly of rigid blocks as stable if and only if all the compressive contact forces between the blocks are positive, and devised an algorithm similar to linear programming for solving the set of contact forces in the assembly. Palmer [21] investigated the computational complexity of stability of polygons and gave definitions of guaranteed stability, potential instability, and infinitesimal stability. Boneschanscher et al. [4] developed a semi-heuristic algorithm to determine the stability of sub-assemblies in an assembly under physical and insertion forces. Mattikalli et al. [17, 16] developed linear programming approaches for identifying the set of orientations under which an assembly is stable in the presence of gravity, both with and without friction. Mosemann et al. [18] developed a method similar to Mattikalli et al. and used it to analyze the stability of the subassemblies at each node of the AND/OR assembly graph [19]. Very little work [19] has used stability analysis systematically for the disassembly process. Moreover, it focuses mainly on the static analysis of the disassembly tree.

The notion of a stable equilibrium is closely related to force closure and form closure in robotic grasping [15, 20] and fixture design for a static assembly [6]. The potential energy minimization principle that governs stability of frictionless assemblies has been used to determine optimum location of fixels for assemblies [27].

\section{B. Geometric Analysis and Planning}

For a concise overview of geometric assembly planning and sequencing, see [12]. The AND/OR graph representation of assembly sequences developed by de Mello and Sanderson [8] enables enumeration of all possible assembly sequences for automated planning. The non-directional blocking graph (NDBG) is a representation that uses the geometry of the parts in the assembly to efficiently determine the set of feasible motion directions for a part [26], thus reducing the combinatorial complexity of disassembly sequencing. The geometric information in the NDBG together with the sequencing information in an assembly AND/OR graph is then used to perform assembly sequencing. Halperin et al. [13] developed a motion space approach to generalize the NDBG to more general part motions.

More recently, disassembly planning has been addressed as a motion planning problem in the composite configuration space of the individual parts by applying samplingbased motion planners for planning part removal paths for disassembly [23]. Techniques have included sampling based on the geometry of reachable configurations. An RRT-based iterative planning approach that gradually decreases the allowed amount of interpenetration has been used for single part disassembly [11]. Le et al. [14] describe an RRT-based method for simultaneous disassembly sequencing and path planning; it can handle nonmonotone disassembly sequences. While these motion planners generate paths that avoid part interference, they do not consider any physical constraints such as gravity, friction, etc.

\section{PROBLEM AND APPROACH}

\section{A. Problem Statement}

The motion stability problem is to identify whether and when instability of an assembly can occur during disassembly part motions. We assume that each part that is moved out is held by a gripper and moved at a constant velocity with perfect position control. The moving part is assumed to undergo quasistatic motion with finite translations. We model the weight of the moving part as being supported by the gripper, unless otherwise stated. Following the standard convention in the assembly planning literature, we do not consider collisions of the gripper with the assembly. We assume frictionless contact unless otherwise stated.

We use linear complementarity [7] to detect whether and when the assembly becomes unstable as each part is removed from the assembly. If linear complementarity finds a solution, i.e., all the contact forces are non-negative and there is no relative motion between parts (excluding the part being moved by the gripper), then the assembly state is considered stable. The linear complementarity problem (LCP) proposed by Baraff [2] for analysis of frictionless cases calculates the relative movement in terms of the relative acceleration between the parts in the assembly. According to Baraff's model, for every contact point $j$ in the assembly, the normal contact acceleration $a_{j}$ and the normal contact force $F_{j}$ form a complementary pair, i.e., $F_{j} \geq 0, \quad a_{j} \geq 0, \quad$ and $\quad F_{j} a_{j}=0$. This statement, which implies that when $F_{j}>0, a_{j}=0$, and when $a_{j}>0, F_{j}=0$, is represented succintly as

$$
0 \leq F_{j} \perp a_{j} \geq 0
$$

We can sum up the complementarity conditions for all $n$ contact points, and since $a_{j}$ and $F_{j}$ are linearly related [1],

$$
\sum_{j=1}^{n} F_{j} a_{j}=\mathbf{F}^{T} \mathbf{a}=\mathbf{F}^{T}(\mathbf{A F}+\mathbf{b})=0
$$

where $\mathbf{A} \in \mathbb{R}^{n \times n}$ is a positive semidefinite matrix. All external and body forces are grouped in the vector $\mathbf{b} \in \mathbb{R}^{n}$.

If friction is included, then in addition to normal forces and accelerations there will be a tangential component of force and acceleration at every contact point. When the static friction force limit is reached at any contact point, the matrix A is no longer positive semidefinite. Baraff's method cannot solve problems when $\mathbf{A}$ is semidefinite. The Stewart-Trinkle complementarity formulation [22] solves contact problems with dynamic friction. In the Stewart-Trinkle model, at each contact point $j$, the complementary constraints are:

$$
\begin{aligned}
0 \leq d_{n_{j}} & \perp \quad p_{n_{j}} \geq 0 \\
0 \leq \rho_{j}=\lambda_{j} e_{j}+W_{f_{j}}^{T} & \perp \quad p_{f_{j}} \geq 0 \\
0 \leq \zeta_{j}=\mu p_{n_{j}}-e_{j}^{T} p_{f_{j}} & \perp \quad \lambda_{j} \geq 0
\end{aligned}
$$

where $d_{n_{j}}$ is the normal distance between the two parts at $j, p_{n_{j}}$ is the normal contact impulse, $W_{f_{j}}$ is the matrix that transforms the generalized velocity $v$ along the edges of the 
friction pyramid approximating the friction cone, $p_{f_{j}}$ is the friction impulse along the edges of the friction pyramid, $e_{j}$ is a vector of ones with size equal to the number of edges of the friction pyramid, $\lambda_{j}$ is a variable that approximates the magnitude of the sliding velocity, and $\mu$ is the Coulomb friction coefficient. For a two-dimensional problem, at each contact point $j$, there are only two edge $\pm t_{j}$ of the friction pyramid, each perpendicular to the outward contact normal $c_{n_{j}}$. The friction contact wrench matrix $W_{f_{j}}$ is

$$
W_{f_{j}}=\left[\begin{array}{cc}
t_{j} & -t_{j} \\
r_{j} \otimes t_{j} & -r_{j} \otimes t_{j}
\end{array}\right]
$$

where $r_{j}$ is the vector from the part centroid to contact point $j$, and $\otimes$ is the two-dimensional equivalent for cross-product.

Using the Stewart-Trinkle model, we formulate the motion path stability problem as a linear complementarity problem:

$0 \leq\left[\begin{array}{ccc}\tilde{\mathbf{A}}_{n n} & \tilde{\mathbf{A}}_{n f} & \mathbf{0} \\ \tilde{\mathbf{A}}_{f n} & \tilde{\mathbf{A}}_{f f} & \tilde{\mathbf{E}} \\ \tilde{\mu} & -\tilde{\mathbf{E}}^{T} & \mathbf{0}\end{array}\right]\left[\begin{array}{c}\tilde{\mathbf{p}}_{n}^{t} \\ \tilde{\mathbf{p}}_{f}^{t} \\ \tilde{\lambda}^{t}\end{array}\right]+\left[\begin{array}{c}\tilde{\mathbf{b}}_{n}^{t} \\ \tilde{\mathbf{b}}_{f}^{t} \\ \mathbf{0}\end{array}\right]=\left[\begin{array}{c}\tilde{\mathbf{d}}_{n}^{t} \\ \Delta t \\ \tilde{\rho}^{t} \\ \tilde{\zeta}^{t}\end{array}\right] \perp\left[\begin{array}{c}\tilde{\mathbf{p}}_{n}^{t} \\ \tilde{\mathbf{p}}_{f}^{t} \\ \tilde{\lambda}^{t}\end{array}\right] \geq 0$

where superscript $t$ indicates value at time $t$. $\tilde{\mathbf{p}}_{n}=$ $\left[p_{n_{1}}, \ldots, p_{n_{K}}\right]^{T}, \quad \tilde{\mathbf{p}}_{f}=\left[p_{f_{1}}, \ldots, p_{f_{K}}\right]^{T}$, and $\tilde{\lambda}^{n}=$ $\left[\lambda_{1}, \ldots, \lambda_{K}\right]^{T}$ are the concatenated normal contact impulses, friction impulses, and $\lambda \mathrm{s}$ respectively for all the $K$ contact points in the assembly, and, $\tilde{\mathbf{A}}_{n n}=\tilde{\mathbf{W}}_{n}^{T} \tilde{\mathbf{M}}^{-1} \tilde{\mathbf{W}}_{n}$, $\tilde{\mathbf{A}}_{n f}=\tilde{\mathbf{W}}_{n}^{T} \tilde{\mathbf{M}}^{-1} \tilde{\mathbf{W}}_{f}, \tilde{\mathbf{A}}_{f f}=\tilde{\mathbf{W}}_{f}^{T} \tilde{\mathbf{M}}^{-1} \tilde{\mathbf{W}}_{f}, \tilde{\mathbf{A}}_{f n}=$ $\tilde{\mathbf{W}}_{f}^{T} \tilde{\mathbf{M}}^{-1} \tilde{\mathbf{W}}_{n}, \tilde{\mathbf{b}}_{f}=\tilde{\mathbf{W}}_{f}^{T} \tilde{\mathbf{M}}^{-1}\left(\tilde{\mathbf{F}}_{b}+\tilde{\mathbf{F}}_{e x t}\right) \Delta t$ and $\tilde{\mathbf{b}}_{n}=$ $\tilde{\mathbf{W}}_{n}^{T}\left(\tilde{\mathbf{M}}^{-1}\left(\tilde{\mathbf{F}}_{b}+\tilde{\mathbf{F}}_{e x t}\right) \Delta t+\tilde{\mathbf{v}}^{t-1}\right)+\tilde{\mathbf{d}}_{n}^{t-1} / \Delta t$.

$\tilde{\mathbf{M}}$ is the concatenated mass matrix, $\tilde{\mathbf{W}}_{n}=\left[W_{n_{1}}, \ldots, W_{n_{K}}\right]$ is the concatenated normal contact wrench matrix where $W_{n_{j}}^{T}=\left[\begin{array}{ll}c_{n_{j}} & r_{j} \otimes c_{n_{j}}\end{array}\right], \tilde{\mathbf{W}}_{f}=\left[W_{f_{1}}, \ldots, W_{f_{K}}\right]$ is the concatenated friction wrench matrix, $\tilde{\mu}=\operatorname{diag}\left[\mu_{1}, \ldots, \mu_{K}\right], \tilde{\mathbf{E}}=$ $\underset{\sim}{\operatorname{diag}}\left[e_{1}, \ldots, e_{K}\right], \tilde{\mathbf{d}}_{n}=\left[d_{n_{1}}, \ldots, d_{n_{K}}\right], \tilde{\rho}=\left[\rho_{1}, \ldots, \rho_{K}\right]$, $\tilde{\zeta}=\left[\zeta_{1}, \ldots, \zeta_{K}\right], \tilde{\mathbf{v}}^{t}$ is the concatenated generalized velocity at time $t$, and $\tilde{\mathbf{F}}_{b}$ and $\tilde{\mathbf{F}}_{\text {ext }}$ are the concatenated body and external forces respectively for all the parts.

After each time step $\Delta t$, we update the position of the moving part, and then use linear complementarity (Equation 2 or Equation 6) to calculate the contact forces and relative movement (acceleration or sliding velocity) of the parts in the assembly.

\section{B. Simulation and Stability Analysis of Disassembly}

We now describe the algorithm to perform stability analysis at each stage of disassembly; it uses linear complementarity for contact force computation, and a path planner to identify motion paths for the parts that are consistent with the NDBG. We describe the approach for evaluating a disassembly sequence for an assembly (see Algorithm 1).

Let $S=<P_{1}, P_{2}, \ldots, P_{N}>$ be the disassembly sequence with the $N$ parts numbered in their disassembly order. Let $A_{N}\left(\bar{q}_{N}\right)$ be the complete assembly comprised of $N$ parts with configuration $\tilde{q}_{N}$, and $A_{N-i}\left(\tilde{q}_{N-i}\right)$ be a subassembly with the first $i$ parts of the sequence removed from the complete assembly. Given part $P_{i}$ at its current configuration $q$, the function MotionPath uses the NDBG and returns a geometrically feasible path $\langle\hat{d}\rangle$ as a sequence of displacement vectors $\hat{d}_{k}$ for $P_{i}$ to move out of the remaining assembly, i.e., $A_{N-i}\left(\tilde{q}_{N-i}\right)$. While $<\hat{d}>$ is nonempty, the next motion step $\hat{d}_{k}$ is executed and the part $P_{i}$ is moved for time $\Delta t$ with a unit velocity along $\hat{d}_{k}$. After $P_{i}$ has moved to its updated position $q$, the function LCPsolve $\left(A_{N-i}\left(\tilde{q}_{N-i}\right), P_{i}(q)\right)$ is called to solve the LCP problem of Equation 2 (for the frictionless case) or Equation 6 (for the case with friction) and compute the contact forces. LCPsolve returns true (i.e., stable) if none of the contact relative motions (accelerations for the frictionless case and velocities for the case with friction) are greater than zero. If any of the contact relative motions are greater than zero, LCPsolve returns false (i.e., unstable) and the simulation is terminated.

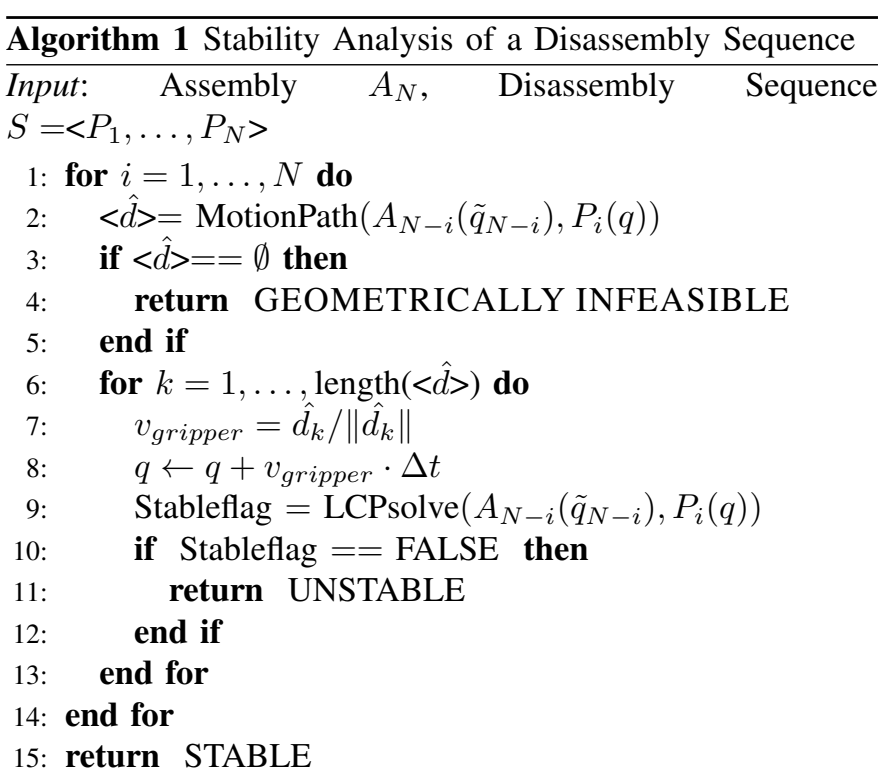

\section{EXAMPLES}

We now consider a few examples. For a specified disassembly sequence, the parts in the assembly are moved along motion paths with directions consistent with the NDBG. At each time step, we calculate the contact forces between different bodies by linear complementarity. If at any time during the simulation, the part that is being moved out by the external gripper induces a relative motion at any contact point in the rest of the assembly, we conclude that the assembly is unstable. Unless otherwise stated the gravitational acceleration $g$ is assumed to be unity, mass density of the blocks is unity, and the simulation time step is $1 \mathrm{sec}$. We will refer to a point $i$ as vertex $i$ if it is a vertex of the body or as a contact point $i$ if it is the corresponding point on the mating body. We will depict the block moved by the gripper in red in the figures. For stability analysis, we use our MATLAB implementation of Baraff's complementarity algorithm for the frictionless case, and for the case with friction, we use a MATLAB interface to the LCPPATH solver [9]. 


\section{A. Simple Example with Blocks}

This example (Figure 1) has a set of frictionless blocks arranged such that if block $\mathrm{C}$ is lifted before block $\mathrm{D}$, block B topples. We use Baraff's complementarity method to analyze this. Consider the disassembly sequence C-D-B-A, with vertical motion of $\mathrm{C}$ (any direction in the upper halfplane above $\mathrm{B}$ is permitted by the NDBG). As C is lifted up, we plot the contact forces, normalized with respect to the weight of moving block $\mathrm{C}$, in Figure 2. At $0 \mathrm{~s}$ all the contact forces are positive. However, at $1 \mathrm{~s}$ when block $\mathrm{C}$ is detached from the rest of the assembly, acceleration at contact point 4 is positive indicating that the rest of the assembly has become unstable. A similar situation occurs when block $\mathrm{C}$ is moved horizontally to the left or right. Thus, all disassembly sequences starting with the removal of block $\mathrm{C}$ are unstable.

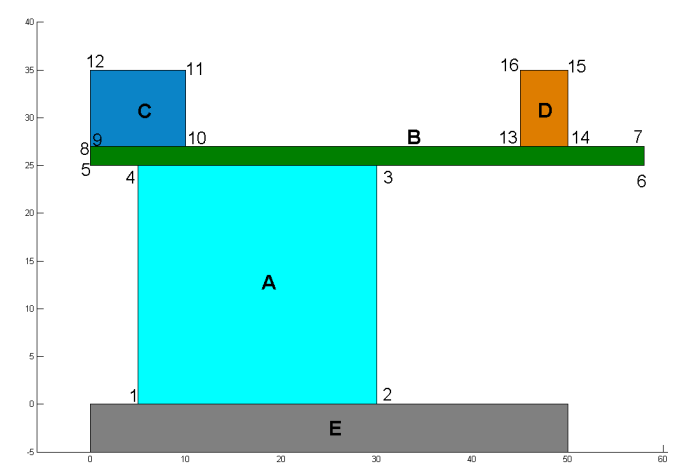

Fig. 1: A simple blocks example: An arrangement of blocks in an equilibrium configuration. The blocks are identified by alphabets, and vertices by numbers. Block $\mathrm{E}$ is the fixed table. Based on [3].

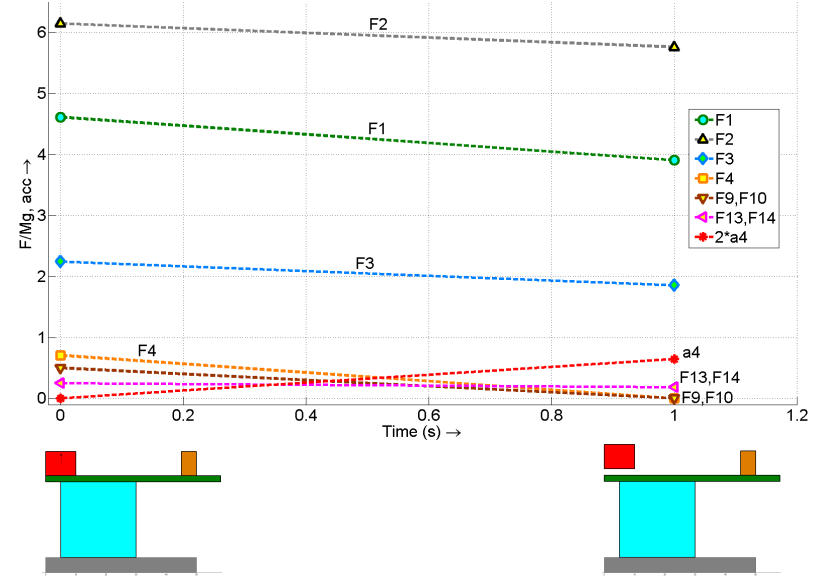

Fig. 2: Plot of contact forces as block $\mathrm{C}$ is lifted up. $\mathrm{X}$-axis is time, Y-axis is contact force normalized by the weight of the moving block $\mathrm{C}$. As soon as $\mathrm{C}$ is lifted up, contact point 4 has positive acceleration indicating that the remaining assembly is unstable.

By similar analyses, we can show that all disassembly sequences that start with the removal of blocks $\mathrm{A}, \mathrm{B}$, or $\mathrm{C}$

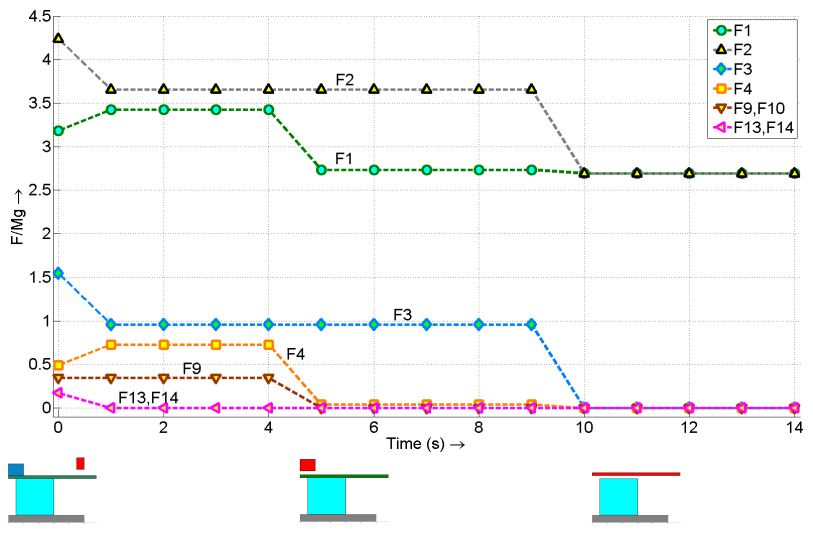

Fig. 3: Plot of contact forces for disassembly sequence D-CB-A. X-axis is time, Y-axis is contact force normalized by the weight of block B. The forces at the contact points of each block remain positive until that block is lifted. This implies that the disassembly sequence is stable.

are unstable. Now consider the sequence D-C-B-A. We show the contact forces (normalized with respect to the weight of the block B) in Figure 3. As each block is lifted up vertically, the contact forces for that particular block become zero. However all other contact forces are positive indicating the remaining subassemblies in all cases are stable. From the complementarity formulation, this implies that the contact accelerations are zero, i.e., none of the contacts are breaking. Hence, D-C-B-A is a stable disassembly sequence, and is in fact the only stable disassembly sequence.

\section{B. Example: Stable Disassembly with and without Fixels}

When disassembly sequences selected using only geometric constraints lead to instability, we can use fixels (i.e., point fixtures) to constrain parts that become unstable as other parts are removed. Using stability analysis, the parts that become unstable at the time of removal of other parts can be identified and stabilized by fixels, or alternative disassembly sequences can be generated.

This example illustrates the use of fixels in maintaining stability of an assembly. Consider blocks A, B, and C on table D (Figure 4). Only block A can move vertically up according to the NDBG (Figure 5). However, as soon as A is lifted up, B becomes unstable. To indicate this we enclose A additionally in a square. However, in this particular example, instability occurs only in B and not in C. To indicate that instability occurs in block B only, we add a red colored arrow from A to B. Now one can identify from the modified NBDG (Figure 5) both the block whose motion causes instability (A), and the one in which instability occurs (B). Similarly, for the horizontal rightward direction, we modify the directional blocking graph (DBG) to indicate the block whose removal causes instability (A), and the block that becomes unstable (B). The instability in B when A is moved out can be prevented by application of fixels at appropriate locations on B.

Figure 6(a) shows the forces at the contact points as $\mathrm{A}$ is 


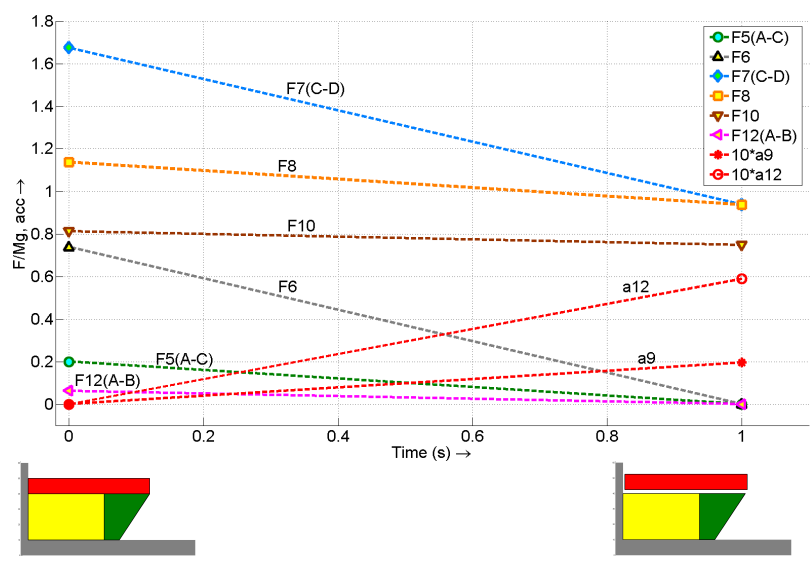

(a)

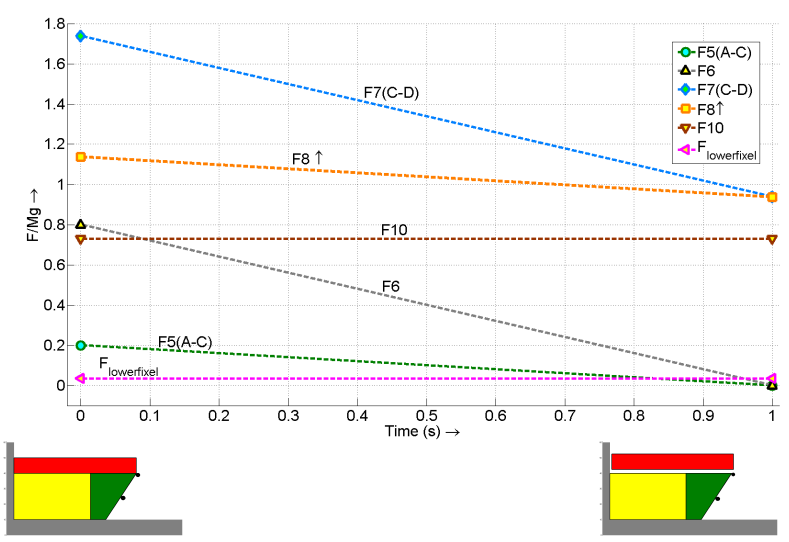

(b)

Fig. 6: Plot of contact forces as block A is lifted up vertically. X-axis is time, Y-axis is contact force normalized by the weight of the moving block A. Note that since there can be more than one normal at a contact vertex, we indicate the bodies between which the contact forces act in the legend of forces and accelerations. (a) Without fixels: As soon as block A is lifted, block $\mathrm{B}$ becomes unstable. This is indicated by positive accelerations at vertices 9 and 12 (scaled by a factor of 10 and shown in red). (b) With fixels: Since the preceding analysis indicates that B loses contact in the horizontal right direction, we put fixels on the right hand free face of $\mathrm{B}$ at vertex 11 and midway between vertices 11 and 10 (as shown in the bottom figures). Now, when A is lifted up, B stays in position, and none of the contact vertices have positive accelerations.

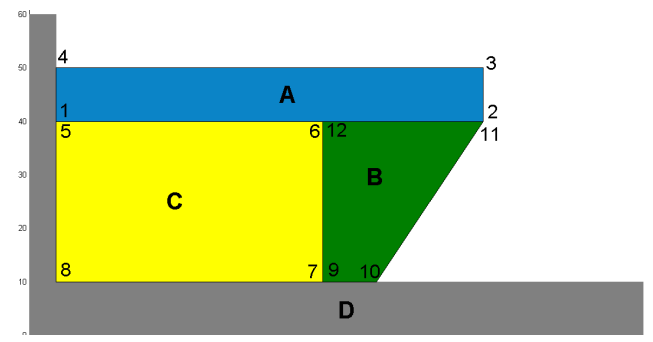

Fig. 4: Three blocks A, B, and C rest on a frictionless base D. The vertices of each movable block are numbered.

lifted up. As soon as A is lifted (at $1 \mathrm{~s}$ ), we note the onset of instability in B at vertices 9 and 12 . The accelerations of 9 and 12 are towards the right, indicating that B will fall in that direction. To prevent instability, we restrict the motion of $\mathrm{B}$ in the horizontal rightward direction by placing fixels at vertex 11 and midway on the edge between vertices 10 and 11 (Figure 6(b)). Now when we lift A up, we see no instability in B. We also note that the fixel at vertex 11 has carried no load throughout the simulation, while the lower fixel supports $\mathrm{B}$ from the start to the end of the simulation. Fixel positioning can be optimized using existing methods, e.g., [27].

\section{The EfFect of Motion Paths on Stability}

We now examine the question: Are part motion paths important in disassembly sequencing? The stability analysis that we have presented so far can alternatively be performed by analyzing the stability of the subassemblies at each node of the assembly sequence AND/OR graph, as in Mosemann et al. [18]. In our work, we simulate the whole process of disassembly by considering part motion. We next demonstrate

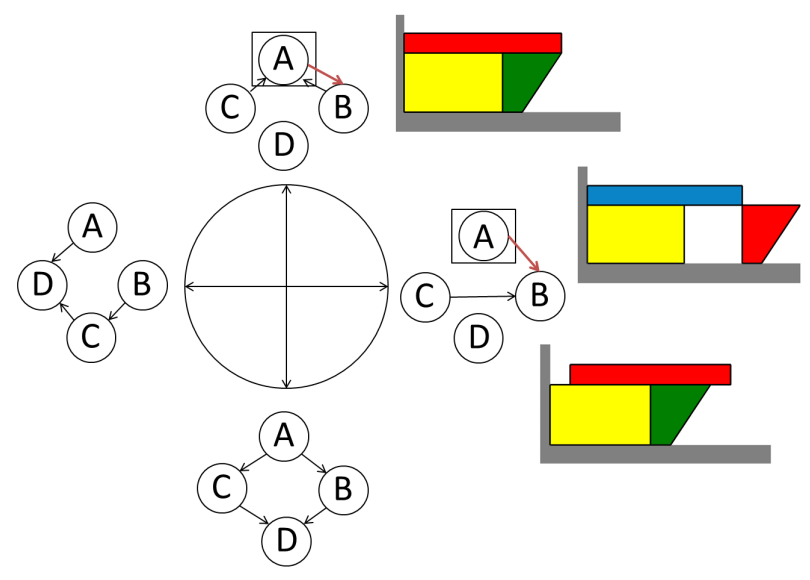

Fig. 5: NDBG for the assembly shown in Figure 4. Case 1: Vertical upward motion (DBG at top). Only block A can be moved. As A is lifted up, it induces instability in block B. The inducer of instability $\mathrm{A}$ is indicated by enclosing it in a square, and a red arrow is drawn from it to the block in which instability occurs, B. Case 2: Horizontal motion to the right (DBG at right). Both blocks A and B can be moved. If B is moved, no instability occurs in the rest of the subassembly. However, if A is moved, it induces instability in B.

that motion paths are also important for determining stable disassembly sequences. We show that instability can occur not only due to the order in which parts are removed, but also depending on the motion paths by which they are removed.

In the example (Figure 7), Blocks A, B, C, and D have to be removed from the part $\mathrm{E}$ that rests on a fixed table (colored grey in Figure 7). The mass densities of blocks $\mathrm{A}$ and $\mathrm{B}$ are 


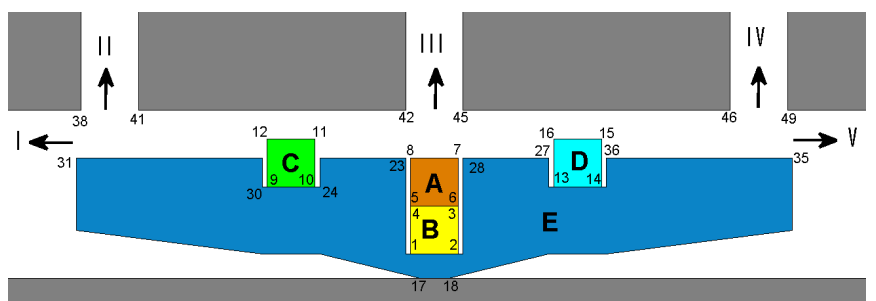

Fig. 7: Example to illustrate the effect of motion paths on stability. A, B, C, D are movable blocks on a nonfixed body E.

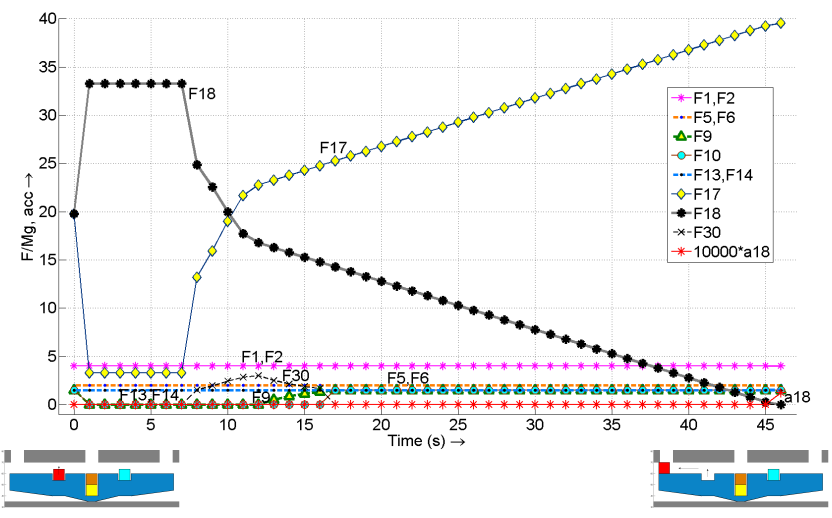

Fig. 8: The effect of motion paths on stability. The Y-axis is contact force normalized by the weight of $\mathrm{C}$. When $\mathrm{C}$ is moved to the left to exit through II at $43 \mathrm{~s}$, no instability is induced. However, when $\mathrm{C}$ is moved to the extreme left towards exit I, at $45 \mathrm{~s}$ the rest of the assembly becomes unstable, as indicated by positive acceleration at vertex 18 .

4 units, those of $\mathrm{C}$ and $\mathrm{D}$ are 3 units, and that of $\mathrm{E}$ is 1 unit. We assume here that the moved part is not supported by the gripper unless in free space. All the blocks are frictionless. The task is to remove blocks A, B, C, and D such that the remaining assembly stays stable. A, B, C, and D can move out of the assembly through exits I, II, III, IV, and V in the top fixed structure (colored grey in Figure 7).

Since this example is symmetric about the vertical axis, we analyze the effect of the motion of block $\mathrm{C}$ to illustrate the influence of motion paths (i.e., choice of exits) on the stability of the assembly. C can be taken out of the assembly through any of its three nearest exits, namely, I, II, and III.

We first illustrate the influence of motion paths on stability. Consider the removal of $\mathrm{C}$ through exit $\mathrm{I}$. As $\mathrm{C}$ is moved to the left through the passage leading to exits I and II, the contact force at vertex 17 increases and that at 18 decreases (Figure 8) to counterbalance the increasing moment produced by the weight of $\mathrm{C}$ as it is moved to the left. At $43 \mathrm{~s}$ when $\mathrm{C}$ is below the passage leading to exit II, it can be moved up towards exit II without instability. However at $45 \mathrm{~s}$ when $\mathrm{C}$ is at exit I, the assembly becomes unstable. This is indicated by positive acceleration at vertex 18 .

When $\mathrm{C}$ is lifted up and moved towards exit III, no instability is induced in the assembly. Thus, removal of $\mathrm{C}$ through exit III is stable. Hence, for disassembly sequences starting with the removal of $\mathrm{C}$, the first step in the disassembly sequence is stable when $\mathrm{C}$ exits through II and III, and not through exit I. A similar situation occurs with D.

Next we illustrate the interplay of motion paths and assembly sequence for stability. Consider removal of A first, followed by C. At $1 \mathrm{~s}$ as A is lifted up and exits through III, the contact forces at vertices 18 and 17 (Figure 9(a)) decrease. Now considering motion of $\mathrm{C}$, at $42 \mathrm{~s}$ when $\mathrm{C}$ is positioned to exit through II, the assembly becomes unstable due to positive acceleration at vertex 18 . Hence, $\mathrm{C}$ cannot exit either through I or II when A has been moved out. Next consider the case when $\mathrm{C}$ is lifted up and moved to the right towards exit III (see Figure 9(b)). As C is moved to the right, the trend in contact forces at vertices 17 and 18 reverses, i.e., contact force decreases at 17 and increases at 18 to counterbalance the moment due to the weight of C. Finally at $37 \mathrm{~s}, \mathrm{C}$ is positioned below exit III, through which it can be moved out. Thus, when A is absent, C can be moved out stably only through exit III.

A similar analysis shows that removal of $\mathrm{C}$ after both $\mathrm{A}$ and $\mathrm{B}$ have been removed always leads to instability.

\section{A. Example: Noninvertibility of Assembly and Disassembly}

When only geometric constraints are considered, a twohanded monotone disassembly can be inverted for assembly [13]. However, when physical forces are considered, a two-handed monotone assembly and disassembly might not be invertible. Although this has been previously mentioned [13], we are not aware of a prior exploration of this issue. Here we construct a simple example to illustrate how a physical force, namely friction, may lead to noninvertibility of assembly and disassembly. The assembly consists of two rigid blocks A and B (Figure 10) that can be disassembled either by lifting up, or by moving horizontally. The disassembly sequence is A-B, and the assembly sequence is B-A. In the vertical direction assembly and disassembly are invertible.

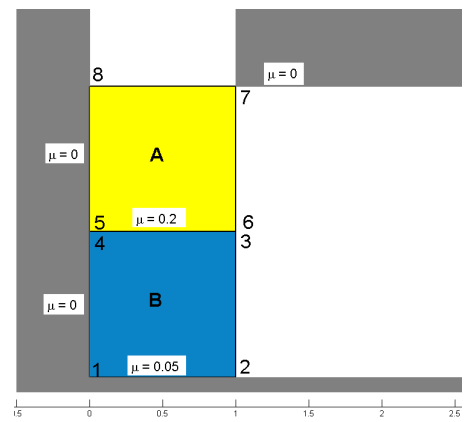

Fig. 10: A noninvertible disassembly example. The blocks can be disassembled either by lifting from the top (invertibly), or by moving horizontally (noninvertibly). The friction coefficients between different surfaces are shown.

Let us now consider disassembly when $\mathrm{A}$ is moved out to the right. We select the coefficients of friction between different surfaces as shown in Figure 10. That is, $\mu$ is 0.2 between A and B, 0.05 between B and its support surface, 


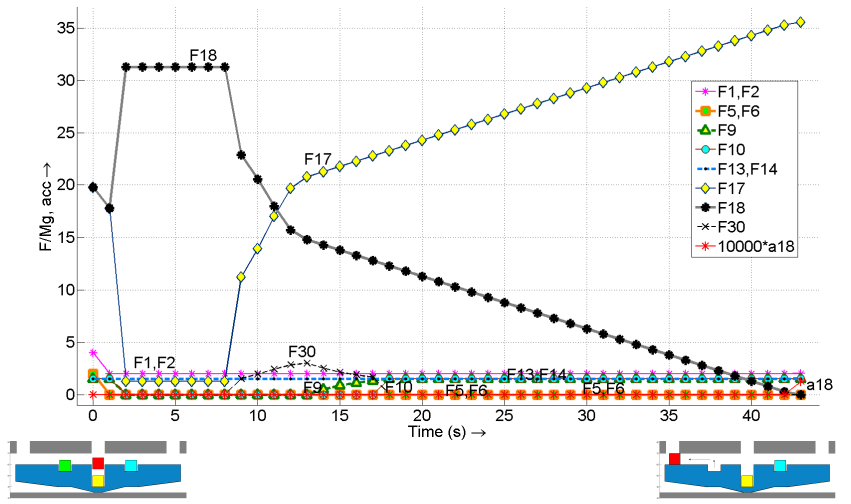

(a)

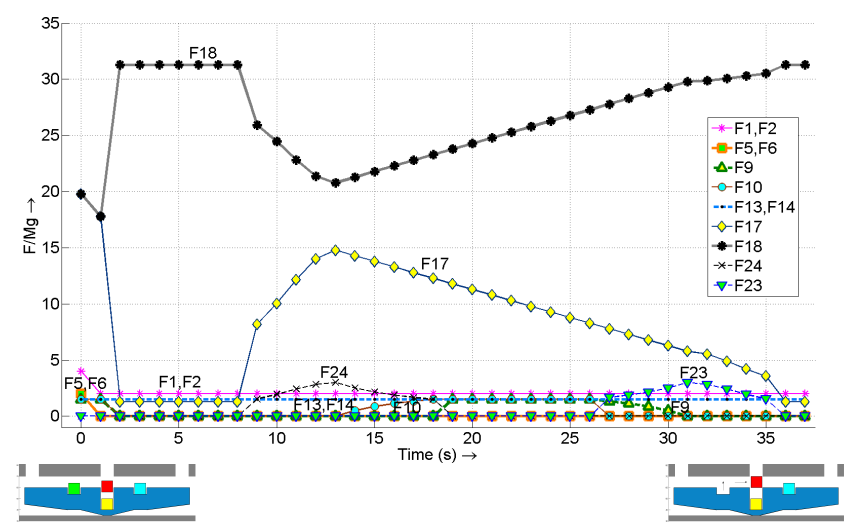

(b)

Fig. 9: The interplay of assembly sequence and motion paths. Y-axis is contact force normalized by the weight of $\mathrm{C}$, and $\mathrm{X}$-axis is time. (a) Block A is first moved out through center exit III. After that, $\mathrm{C}$ is moved to the left. At $42 \mathrm{~s}$, before $\mathrm{C}$ is in position to exit through II, the assembly becomes unstable. Thus, when A is removed first, $\mathrm{C}$ cannot exit through either of exits I and II. (b) A is moved out first and then C is then moved to exit III. In this case the assembly remains stable.

and zero between all other surfaces. The weight of each block is one unit. The complementarity conditions for this system are given by Equation 6, and we use the LCPPATH solver to calculate the forces between different parts in the assembly (Figure 11). When $\mathrm{A}$ is moved to the right by applying a force, it causes B to slide to the right. When a force high enough to move A is applied, it begins to slide, and the friction force at the bottom is 0.2 units (towards the left). This force also acts on B in the opposite direction. However the friction force between $\mathrm{B}$ and the base is only 0.1 units. This imbalance of friction forces between the top and bottom surfaces of B causes B to slide to the right in the same direction as A. Since movement of A induces movement in $\mathrm{B}$, the disassembly sequence is unstable. If we invert this disassembly sequence, then the assembly sequence is B-A. When A is inserted horizontally over B from the right, the sliding motion of B is prevented by the left wall, and hence it is stable. Thus, when the horizontal path is selected, assembly B-A and disassembly A-B are not invertible.

While the main objective of this example is to demonstrate the noninvertibility of assembly and disassembly in the presence of friction, this example also illustrates the effect of the motion path on disassembly. For the vertical path, assembly and disassembly are invertible, whereas they are not invertible for the horizontal path.

\section{CONCLUSION}

The focus of this paper is the influence of part motion path and assembly sequence on assembly stability. Given this goal, we go beyond the prior approach of performing a static analysis of the disassembly tree. We instead use linear complementarity to perform the stability analysis at each step of motion during the disassembly process as parts are removed sequentially. We show that disassembly sequences that are consistent with only geometric contraints may be infeasible in the presence of gravity or friction. Furthermore,

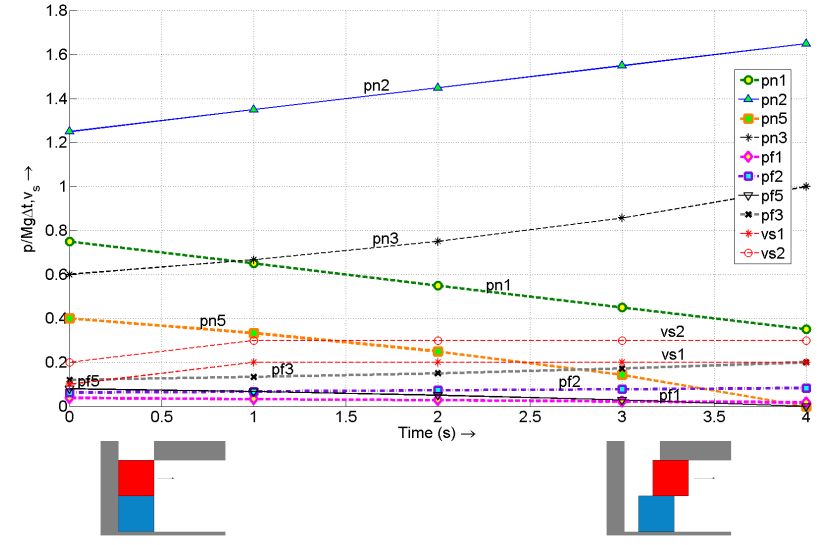

Fig. 11: Plot of normal contact and friction impulses when A is moved to the right, normalized by the normal impulse of A. The normal contact impulses are indicated by subscript $n$ and the friction impulses by subscript $f$. The sliding velocity between $\mathrm{A}$ and $\mathrm{B}$ is $v s_{2}$ and that between $\mathrm{B}$ and the base is $v s_{1}$.

stability analysis can be used to identify and prevent instability inducing motions by introducing fixtures or by selecting alternative disassembly sequences. Previously, only the disassembly sequence had been shown to be important for stable disassembly. We have shown that not only the disassembly sequence, but the motion paths of the parts can also affect the stability of the disassembly process. We also show using a simple example that assembly and disassembly, invertible when only geometric constraints are considered, can become noninvertible in the presence of physical forces. Future work will focus on integrating the stability analysis with motion planning of the parts. Our approach can be extended to 3D parts, and we will explore application to industrial examples with complex geometry. 


\section{ACKNOWLEDGMENTS}

This work was supported in part by NSF Awards CCF0729161, IIS-1019160, and CCF-1048261. Thanks to Nilanjan Chakraborty and Raju Mattikalli for informative discussions, and the reviewers for helpful comments.

\section{REFERENCES}

[1] D. Baraff. Analytical methods for dynamic simulation of nonpenetrating rigid bodies. ACM SIGGRAPH Computer Graphics, 23(3):223-232, 1989.

[2] D. Baraff. Fast contact force computation for nonpenetrating rigid bodies. In Annual Conference on Computer Graphics and Interactive Techniques (SIGGRAPH '94), pages 23-34, 1994.

[3] M. Blum, A. Griffith, and B. Neumann. A stability test for configurations of blocks. Technical Report AI Memo. 188, Massachusetts Institute of Technology, Feb. 1970.

[4] N. Boneschanscher, H. van der Drift, S. J. Buckley, and R. H. Taylor. Subassembly stability. In National Conference on Artificial Intelligence, pages 780-785, 1988.

[5] G. Boothroyd and P. Dewhurst. Assembly Automation and Product Design. Taylor and Francis, New York, 2005.

[6] R. C. Brost and K. Y. Goldberg. A complete algorithm for designing planar fixtures using modular components. IEEE Transactions on Robotics and Automation, 12(1):31-46, Feb. 1996.

[7] R. W. Cottle, J. S. Pang, and R. E. Stone. The Linear Complementarity Problem. Academic Press, Boston, MA, 1992.

[8] L. S. H. de Mello and A. C. Sanderson. AND/OR graph representation of assembly plans. IEEE Transactions on Robotics and Automation, 6(2):188-199, Apr. 1990.

[9] S. P. Dirkse and M. C. Ferris. The PATH solver: A non-monotone stabilization scheme for mixed complementarity problems. Optimization Methods and Software, 5:123-156, 1995.

[10] C. Erdogan and M. Stilman. Planning in constraint space: Automated design of functional structures. In IEEE International Conference on Robotics and Automation, pages 1799-1804, Karlsruhe, Germany, May 2013.

[11] E. Ferré and J. P. Laumond. An iterative diffusion algorithm for part disassembly. In IEEE International Conference on Robotics and Automation, pages 31493154, New Orleans, LA, Apr. 2004.

[12] D. Halperin, L. Kavraki, and J.-C. Latombe. Robotics. In J. E. Goodman and J. O'Rourke, editors, Handbook of Discrete and Computational Geometry, pages 10651093. Chapman and Hall/CRC, Boca Raton, Florida, second edition, 2004.

[13] D. Halperin, J. C. Latombe, and R. H. Wilson. A general framework for assembly planning: The motion space approach. Algorithmica, 26(3-4):577-601, 2000.

[14] D. T. Le, J. Cortes, and T. Simeon. A path planning approach to (dis)assembly sequencing. In IEEE Interna- tional Conference on Automation Science and Engineering, pages 286-291, Bangalore, India, Aug. 2009.

[15] M. T. Mason. Mechanics of Robotic Manipulation. MIT Press, Cambridge, MA, 2001.

[16] R. Mattikalli, D. Baraff, and P. Khosla. Finding all stable orientations of assemblies with friction. IEEE Transactions on Robotics and Automation, 12(2):290301, Apr. 1996.

[17] R. Mattikalli, D. Baraff, P. Khosla, and B. Repetto. Gravitational stability of frictionless assemblies. IEEE Transactions on Robotics and Automation, 11(3):374388, June 1995.

[18] H. Mosemann, F. Rohrdanz, and F. M. Wahl. Stability analysis of assemblies considering friction. IEEE Transactions on Robotics and Automation, 13(6):805813, 1997.

[19] H. Mosemann, F. Rohrdanz, and F. M. Wahl. Assembly stability as a constraint for assembly sequence planning. In IEEE International Conference on Robotics and Automation, pages 233-238, Leuven, Belgium, May 1998.

[20] R. M. Murray, Z. Li, and S. S. Sastry. A Mathematical Introduction to Robotic Manipulation. CRC Press, Boca Raton, FL, 1994.

[21] R. S. Palmer. Computational Complexity of Motion and Stability of Polygons. $\mathrm{PhD}$ thesis, Cornell University, Ithaca, NY, 1987.

[22] D. E. Stewart and J. C. Trinkle. An implicit timestepping scheme for rigid body dynamics with inelastic collisions and Coulomb friction. International Journal of Numerical Methods in Engineering, 39:2673-2691, 1996.

[23] S. Sundaram, I. Remmler, and N. M. Amato. Disassembly sequencing using a motion planning approach. In IEEE International Conference on Robotics and Automation, pages 1475-1480, Seoul, Korea, May 2001.

[24] J. Wang, P. Rogers, L. Parker, D. Brooks, and M. Stilman. Robot Jenga: Autonomous and strategic block extraction. In IEEE/RSJ International Conference on Intelligent Robots and Systems, pages 5248-5253, St. Louis, MO, Oct. 2009.

[25] D. E. Whitney. Mechanical Assemblies: Their Design, Manufacture, and Role in Product Development. Oxford University Press, 2004.

[26] R. H. Wilson and J.-C. Latombe. Geometric reasoning about mechanical assembly. Artificial Intelligence, 71(2):371-396, Dec. 1994.

[27] J. D. Wolter and J. C. Trinkle. Automatic selection of fixture points for frictionless assemblies. In IEEE International Conference on Robotics and Automation, volume 1, pages 528-534, San Diego, CA, May 1994. 